\title{
Descrição do processo xenarthro em Bradypus variegatus
}

\author{
[Description of the xenarthro process in Bradypus variegatus]
}

\section{"Artigo Científico/Scientific Article"}

\author{
Priscilla Vírginio de Albuquerque ${ }^{1 *}$, Marleyne José Afonso Accioly Lins Amorim ${ }^{1}$, Lucilo Bioni da
} Fonsêca Filho $^{2}$, Silvia Fernanda de Alcantara ${ }^{3}$, Maria Eduarda Luiz Coelho de Miranda ${ }^{3}$, Gilcifran Prestes de Andrade ${ }^{1}$, Júlio Cézar dos Santos Nascimento ${ }^{4}$

\begin{abstract}
${ }^{1}$ Departamento de Morfologia e Fisiologia Animal, Universidade Federal Rural de Pernambuco, Recife-PE, Brasil.
${ }^{2}$ Departamento de Medicina Veterinária, Universidade Federal Rural de Pernambuco, Recife-PE, Brasil.

${ }^{3}$ Departamento de Biologia, Universidade Federal Rural de Pernambuco, Recife-PE, Brasil.

${ }^{4}$ Departamento de Zootecnia, Universidade Federal Rural de Pernambuco, Recife-PE, Brasil.

*Autor para correspondência/Corresponding author: E-mail: priscilla2009w@ hotmail.com
\end{abstract}

\section{Resumo}

Tamanduás, bichos-preguiça e tatus são considerados xenarthras, devido a possuírem articulações adicionais nas vértebras denominadas de xenarthro, justificando a Superordem. Estas articulações permitem-lhes assumir uma postura ereta sobre um tripé formado pelos membros posteriores e a cauda. No entanto, a determinação desses processos vertebrais no bicho-preguiça é inexistente na literatura. Devido à importância do conhecimento das estruturas ósseas dos animais para o entendimento de seus hábitos, propôs-se descrever o processo xenarthro na preguiça Bradypus variegatus. Foram utilizados três esqueletos, sendo um jovem e dois adultos pertencentes ao acervo do Museu de Anatomia Comparada da Universidade Federal Rural de Pernambuco (MAC-UFRPE). Realizou-se uma análise macroscópica das vértebras das preguiças. Pode-se descrever os xenarthros como processos presentes na última vértebra torácica, em todas as vértebras lombares, e na primeira vértebra sacral, medindo em média $0,5 \mathrm{~cm}$, sendo localizado ventralmente ao processo mamilar formando com este uma concavidade, onde se articula a base do processo transverso da vértebra anterior e é este encaixe diferenciado que garante uma maior estabilidade ao animal.

Palavras-chave: osteologia; processos articulares; vértebras.

\begin{abstract}
Anteaters, sloths and armadillos are considered xenarthrans, because they have additional joints in the vertebrae called xenarthrous, justifying the superorder. These joints allow them to stand upright on a tripod formed by their hind limbs and tail. However, the determination of these vertebral processes in the sloth is not available in the literature. Due to the importance of knowledge of the bone structures of animals to understand their habits, it was proposed to describe the xenarthrous process in sloth Bradypus variegatus. Three skeletons were used, one young and two adults from the collection of the Museum of Comparative Anatomy of the Federal Rural University of Pernambuco (MAC-UFRPE). A macroscopic analysis of the sloth vertebrae was performed. The xenarthrous can be described as processes present in the last thoracic vertebra, in all lumbar vertebrae, and in the first sacral vertebra, measuring on average $0.5 \mathrm{~cm}$, being located ventrally to the mamillary process forming a concavity, where the base of the transverse process of the anterior vertebra articulates. It is this differentiated fitting that ensures greater stability to the animal.
\end{abstract}

Keywords: articular process; osteology; vertebrae.

\section{Introdução}

A Superordem Xenarthra, formada por preguiças, tamanduás e tatus (Wetzel, 1982), possui uma característica osteológica importante, que é a presença de um processo articular ou 
anapófise que se articula ventralmente com as metapófises, ou entre elas e os processos transversos (Rose, 2006). Estas articulações permitem que estes animais assumam uma postura ereta, formando um tripé sobre os membros posteriores em conjunto com a cauda, tal posicionamento está associado à defesa, observação do ambiente e obtenção de alimentos (Wetzel, 1982).

A coluna vertebral nos mamíferos apresenta uma grande variabilidade, reflexo dos distintos ambientes onde este grupo se adaptou (Galis et al., 2014). Apesar disto, alguns táxons mantiveram pouca variação, quanto à quantidade de vértebras e suas características (Sánchez-Villagra, 2010). As exceções são as Superodem Afrotheria e Xenarthra, esta última possui uma quantidade variável de vértebras tóraco-lombares entre as espécies que a compõe (Sánchez-Villagra et al., 2007).

Nos mamíferos, a sucessão vertebral não é unida apenas pelos discos intervertebrais, há um par simples de articulações sinoviais, com a presença de processos articulares denominados zigapófises, presentes no arco neural. Em adição a estas articulações os xenarthras possuem um ou mais pares de articulações intervertebrais suplementares, presente em todas as vértebras lombares e em número variável nas torácicas (Gaudin e Biewener, 1992). Estas articulações supranumerárias são tituladas de xenarthrales (Gaudin e Biewener, 1992) ou processo xenarthro (Endo et al., 2009) e são bem descritas no tatugalinha (Dasypus novemcinctus) (Gaudin e Biewener, 1992) e no tamanduá-bandeira (Myrmecophaga tridactyla) (Endo et al., 2009), porém na literatura, a determinação destes processos vertebrais em bichos-preguiça é inexistente.

Tendo em vista a importância do conhecimento destas estruturas ósseas nestes animais, para fins médicos veterinários, o objetivo deste estudo é descrever o processo xenarthro ou xenarthrales em bicho-preguiça (Bradypus variegatus).

\section{Material e Métodos}

Foram utilizados cinco bichos-preguiça da espécie $B$. variegatus, sendo três esqueletos, um de animal jovem e dois de animais adultos, pertencentes ao acervo do Museu de Anatomia Comparada da Universidade Federal Rural de Pernambuco (MAC-UFRPE).
Com os esqueletos foi feita uma observação macroscópica das vértebras das preguiças, a fim de identificar os processos xenarthros, que foram medidos com paquímetro manual. As análises realizadas foram descritas e fotografadas com auxílio de uma câmera Canon T6. E as denominações das estruturas foram baseadas nas determinações da Nomina Anatômica Veterinária de 2017.

\section{Resultados e Discussão}

Pode-se descrever o xenarthro como um processo vertebral localizado ventralmente ao processo mamilar, formando com este uma concavidade, que se articula com a base do processo transverso da vértebra anterior e é esta junção diferenciada que garante maior estabilidade ao animal. Foi observado que o processo xenartho, nas preguiças, mede em média $0,5 \mathrm{~cm}$ e conta com apenas uma face articular (Figuras 1 e 2).

Foi identificada a presença deste processo na última vértebra torácica, em todas as vértebras lombares, e na primeira vértebra sacral de todos os esqueletos analisados.

Segundo este estudo, a face articular dos processos xenarthros, bem como a face articular dos processos mamilares de cada vértebra, se articulam na face ventral e dorsal, respectivamente, da base do processo transverso da vértebra anterior (Figuras 1 e 2). Este encaixe é similar entre as vértebras, torácica, lombar e sacral. Durante a extensão da coluna vertebral o processo xenarthro fica bem inserido no processo transverso, enquanto durante a flexão da mesma, esta inserção é diminuída.

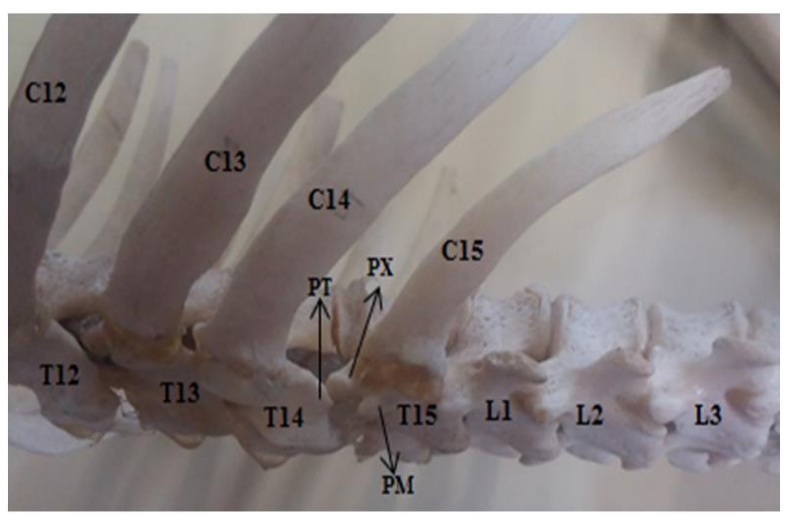

Figura 1. Fotomacroscopia com vista lateral de parte da coluna vertebral do bicho-preguiça, indicando o processo xenarthro (PX). Costelas $(\mathrm{C})$, vértebras torácicas $(\mathrm{T})$, vértebras lombares $(\mathrm{L})$, processo transverso (PT), processo mamilar (PM). 


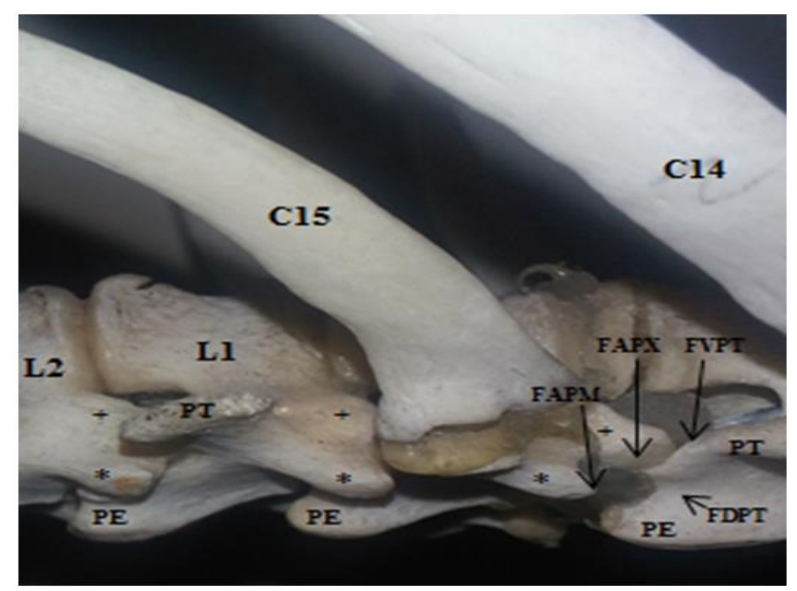

Figura 2. Fotomacroscopia com vista lateral de parte da coluna vertebral do bicho-preguiça, evidenciando a articulação vertebral adicional desses animais. Costelas (C), vértebras lombares (L), processo espinhoso (PE), processo transverso (PT), face articular do processo xenarthro (FAPX), face articular do processo mamilar (FAPM), face ventral do processo transverso (FAPT), face dorsal do processo transverso (FDPT), processo xenarthro $(+)$, processo mamilar $(*)$.

Os representantes da superordem Xenarthra caracterizam-se por uma significativa variação no número de vértebras, podendo ocorrer até mesmo entre indivíduos da mesma espécie, como acontece com as vértebras cervicais da preguiça $(B$. variegatus) (Nowak, 1999; Asher et al., 2011). Neste contexto, o processo xenarthro apresenta diferentes localizações dependendo do xenarthra, no caso do tamanduá-bandeira está nas cinco últimas torácicas e nas três primeiras lombares (Endo et al., 2009). Enquanto que nos bichospreguiça foi encontrado em vértebra torácica, lombar e sacral.

Estes processos vertebrais adicionais participam da composição da articulação sinovial existente entre as vértebras, assim como os processos articulares craniais e caudais. Tal articulação é caracterizada pela presença de um disco e uma série de ligamentos que prendem os corpos e os arcos vertebrais (Getty, 1986). Segundo Gaudin e McDonald (2008), essas anapófises são pouco desenvolvidas nas preguiças atuais, nem sendo vistas em todos os representantes do gênero Choloepus (Kraft, 1995). E teriam surgido como uma adaptação a hábitos fossoriais de um possível ancestral de todos os xenarthras (Nowak, 1999; Rose, 2006; Endo et al., 2009). Acredita-se que a presença do processo xenarthro no tatu-galinha $(D$. novemcinctus) seja crucial para que esta espécie suporte o peso corporal nos membros torácicos e pélvicos, com o objetivo de manter a marcha e a flexibilidade. Para os tamanduás, garante uma liberdade dos membros torácicos, uma vez que possibilita que o peso da parte superior do corpo seja suportado pelos membros pélvicos, favorecendo os hábitos alimentares e de defesa (Gaudin e Biewener, 1992). No caso do bichopreguiça (B. variegatus) a presença deste processo faz com que estes animais possam ter uma postura ereta contribuindo para seu hábito arborícola (Wetzel, 1982).

\section{Conflito de Interesse}

Os autores declaram não existir conflito de interesse.

\section{Comissão de Ética}

O trabalho foi aprovado sob a licença $n^{\circ}$ 034/2015 do Comitê de Ética no Uso de Animais da Universidade Federal Rural de Pernambuco UFRPE. E autorização Sisbio-ICMBio nº 46665-3.

\section{Agradecimentos}

A equipe do Museu de Anatomia Comparada MAC-UFRPE pela disponibilidade dos esqueletos para o estudo.

\section{Referências}

Asher, R.J.; Lin, K.H.; Kardjilov, N.; Hautier, L. Variability and constraint in the mammalian vertebral column. Journal of Evolutionary Biology, 24(5): 1080-1090, 2011.

Endo, H.; Komiya, T.; Kawada, S.; Hayashida, A.; Kimura, J.; Itou, T.; Koie, H.; Sakai, T. Threedimensional reconstruction of the xenarthrous process of thoracic and lumber vertebrae in the giant anteater. Mammal Study, 34(1):1-6, 2009.

Galis, F.; Carrier, D.R.; Van Halpen, J.; Van Der Mije, S.D.; Van Dooren, T.J.; Metz, J.A.J.; Ten Broek, C.M.A. Fast running restribcts evolutionary change of the vertebral column in mammals. Proceedings of the National Academy of Sciences of the United States of America, 111(31):11401-11406, 2014.

Gaudin, T.J.; Biewener, A.A. The functional morphology of xenarthrous vertebrae in the armadillo Dasypus novemcinctus (Mammalia Xenarthra). Journal of Morphology, 214(1): 63-81, 1992.

Gaudin, T.J.; McDonald, H.G. Morphology-based investigations of the phylogenetic relationships among extant and fossil xenarthrans. In: Vizcaíno, S.F.; Loughry, W.J. The biology of the xenarthra. $1^{\text {st. }} \mathrm{ed}$. 
Gainesville: University Press of Florida, 2008. p.24-36.

Getty, R. Sisson \& Grossman - anatomia dos animais domésticos. $5^{\text {a }}$ ed. v.1. Rio de Janeiro: Editora Guanabara Koogan, 1986. $1134 \mathrm{p}$.

Kraft, R. Xenarthra. In: Niethammer, J.; Schlieman, H.; Starck, D. (Ed.). Handbuch der zoologie. Mammalia. v. $8.1^{\text {st }}$ ed. Berlin: de Gruyter \& Co, 1995, p. 1-79.

Nowak, R.M. Walker's mammals of the world. $6^{\text {nd }}$ ed., Baltimore: The Johns Hopkins University Press, 1999, 1936p.

Rose, K.D. The beginning of the age of mammals. ${ }^{\text {st }}$ ed. Baltimore: Johns Hopkins University Press, 2006. 448p.

Sánchez-Villagra, M.R. Developmental palaentology in synapsids: the fossil record of ontogeny in mammals and their closest relatives. The Royal Society, 277(1685):1139-1147, 2010.

Sánchez-Villagra, M.R.; Narita, Y.; Kuratani, S. Thoracolumbar vertebral number: the first skeletal synapomorphy for afrotherian mammals. Systematics and Biodiversity, 5(1):1-7, 2007.

Wetzel, R.M. Systematics, distribution, ecology, and conservation of South American Edentates. In: Mares, M.A.; Genoway, H.H. (Ed.). Mammalian biology in South America: a symposium held at the Pymatuning Laboratory of Ecology. $1^{\text {st }}$ ed. Linesville: Pymatuning Laboratory of Ecology, 1982. p. 345-375. 